\title{
RadMAP: The Radiological Multi-sensor Analysis Platform
}

\author{
Mark S. Bandstra ${ }^{\mathrm{a}, *}$, Timothy J. Aucott ${ }^{\mathrm{b}}$, Erik Brubaker ${ }^{\mathrm{c}}$, Daniel H. Chivers ${ }^{\mathrm{a}}$, \\ Reynold J. Cooper ${ }^{\mathrm{a}}$, Joseph C. Curtis ${ }^{\mathrm{a}, \mathrm{b}}$, John R. Davis ${ }^{\mathrm{b}}$, Tenzing H. Joshi ${ }^{\mathrm{a}}$, \\ John Kua ${ }^{\mathrm{a}}$, Ross Meyer ${ }^{\mathrm{a}}$, Victor Negut ${ }^{\mathrm{a}}$, Michael Quinlan ${ }^{\mathrm{a}}$, Brian J. Quiter ${ }^{\mathrm{a}}$, \\ Shreyas Srinivasan ${ }^{\mathrm{b}, \mathrm{d}}$, Avideh Zakhor ${ }^{\mathrm{d}}$, Richard Zhang ${ }^{\mathrm{d}}$, Kai Vetter ${ }^{\mathrm{a}, \mathrm{b}}$ \\ ${ }^{a}$ Nuclear Science Division, Lawrence Berkeley National Laboratory, Berkeley, California \\ ${ }^{b}$ Department of Nuclear Engineering, University of California Berkeley, California \\ ${ }^{c}$ Sandia National Laboratory, Livermore, California \\ ${ }^{d}$ Department of Electrical Engineering and Computer Science, University of California \\ Berkeley, California
}

\begin{abstract}
The variability of gamma-ray and neutron background during the operation of a mobile detector system greatly limits the ability of the system to detect weak radiological and nuclear threats. The natural radiation background measured by a mobile detector system is the result of many factors, including the radioactivity of nearby materials, the geometric configuration of those materials and the system, the presence of absorbing materials, and atmospheric conditions. Background variations tend to be highly non-Poissonian, making it difficult to set robust detection thresholds using knowledge of the mean background rate alone. The Radiological Multi-sensor Analysis Platform (RadMAP) system is designed to allow the systematic study of natural radiological background variations and to serve as a development platform for emerging concepts in mobile radiation detection and imaging. To do this, RadMAP has been used to acquire extensive, systematic background measurements and correlated contextual data that can be used to test algorithms and detector modalities at low false alarm rates. By combining gamma-ray and neutron detector systems with data from contextual sensors, the system enables the fusion of data from multiple sensors
\end{abstract}

\footnotetext{
* Corresponding author

Email address: msbandstra@lbl.gov (Mark S. Bandstra)
}

Preprint submitted to Nuclear Instruments and Methods in Physics A September 17, 2016 
into novel data products. The data are curated in a common format that allows for rapid querying across all sensors, creating detailed multi-sensor datasets that are used to study correlations between radiological and contextual data, and develop and test novel techniques in mobile detection and imaging. In this article we will describe the instruments that comprise the RadMAP system, the effort to curate and provide access to multi-sensor data, and some initial results on the fusion of contextual and radiological data.

Keywords: Gamma-ray detection, Gamma-ray imaging, Background radiation, Data fusion, Homeland security, Radiological search

\section{Introduction}

Searching for radioactive sources in urban environments is an important component of modern radiological and nuclear security. Toward this end, various vehicle-borne, large area detector systems have been designed and tested in re5 cent years [1-4. However, the problem of detecting illicit radioactive sources is confounded by the non-Poissonian background fluctuations commonly encountered in operational environments, which greatly limit the capabilities of these systems [5] 8 . These fluctuations are caused by variations in the environment through which the vehicle is moving, but the exact radiological properties and geometric configuration of materials around the vehicle are seldom known well enough to predict or even understand these changes.

Many techniques have been brought to bear on the problem of background fluctuations in mobile detector systems. For systems using spectroscopic detectors, new finely tuned spectroscopic algorithms have been developed in recent years to account for background variations 9 -17]. Some systems use some basic form of imaging, such as passive or active coded masking, whereby some source counts are sacrificed for improved knowledge of the background [2 4, 18, 19].

The Radiological Multi-sensor Analysis Platform (RadMAP) system has been developed to serve both as a unique experimental test bed for new concepts in mobile radiation detection and imaging, as well as a platform for the acquisi- 
tion of the large, diverse multi-sensor datasets required for the systematic study of factors affecting detection performance in real-world environments. The data acquired with the RadMAP system may be used to facilitate, for example:

- The systematic study of natural radiological background variations across diverse environments.

- The investigation of correlations between radiological background and contextual information.

- The development of novel algorithms for detection, imaging, and multisensor fusion.

- The characterization and performance assessment of algorithms at low false alarm rates.

- Trade studies of the factors influencing the design and implementation of mobile detection systems.

The broader scientific community has been given access, upon request, to the AP data to allow such studies to be performed. Access to the multisensor data is provided through the LBNL developed Gamma-Ray Data Cloud (GRDC). General access to the multi-sensor data is provided through the LBNLdeveloped Gamma-Ray Data Cloud (GRDC).

In this paper, we will describe the RadMAP system and its suite of radiological and contextual sensors, and discuss several initial studies that have been performed using the data that it has collected. The possibilities for multisensor data fusion and the data service used to distribute the data will also be discussed.

\section{RadMAP overview}

45 The RadMAP vehicle was originally the Mobile Imaging and Spectroscopic Threat Identification (MISTI) vehicle built by the Naval Research Laboratory [2, 20, for the Domestic Nuclear Detection Office's Standoff Radiation Detection System (SORDS) program. MISTI used a combination of high purity 
germanium (HPGe) detectors for spectroscopic alarming and isotope identification, and an array of sodium iodide $(\mathrm{NaI}(\mathrm{Tl}))$ detectors with a lead coded mask for imaging. In addition, it had a side-facing camera, a multi-antenna Global Positioning System (GPS) receiver, and software for real-time gamma-ray source imaging and localization.

RadMAP represents a further development of MISTI's gamma-ray detectors and environmental sensors to include panoramic video, Light Detection And Ranging (LiDAR), weather, hyperspectral, and other sensors in order to provide a context for the radiation measurements that is as complete as possible (see Section 3). The HPGe detectors have been upgraded from liquid-nitrogen cooling to mechanical cooling to make longer measurement campaigns possible,

60 and liquid-scintillator detectors have been added to enable the same explorations in the fast neutron domain.

As of 2016, RadMAP has traveled approximately 4000 miles in the San Francisco Bay Area, including through many urban areas such as Berkeley, Oakland, and San Francisco, in addition to some suburban and rural areas.

${ }_{65}$ The areas surveyed by the system to date are shown in Figure 1 .

\section{RadMAP instruments}

\subsection{Radiation detectors}

The core sensors of the RadMAP vehicle consist of three types of radiation detectors: a $10 \times 10$ array of $\mathrm{NaI}(\mathrm{Tl})$ detectors with a coded lead mask for imaging, an array of between 14 and 24 HPGe detectors, and an array of 16 liquid scintillators for fast neutron detection. All of these detector systems report events in list mode; i.e., individual events are stored with timestamps. A cutaway view of the vehicle with the placements of these sensors is shown in Figure 2 .

75 3.1.1. $\mathrm{NaI}(\mathrm{Tl})$ array

The gamma-ray imager aboard RadMAP consists of $1004 \times 4 \times 2$ inch NaI(Tl) detectors arranged in a $10 \times 10$ configuration, totaling approximately $1 \mathrm{~m}^{2}$ of 


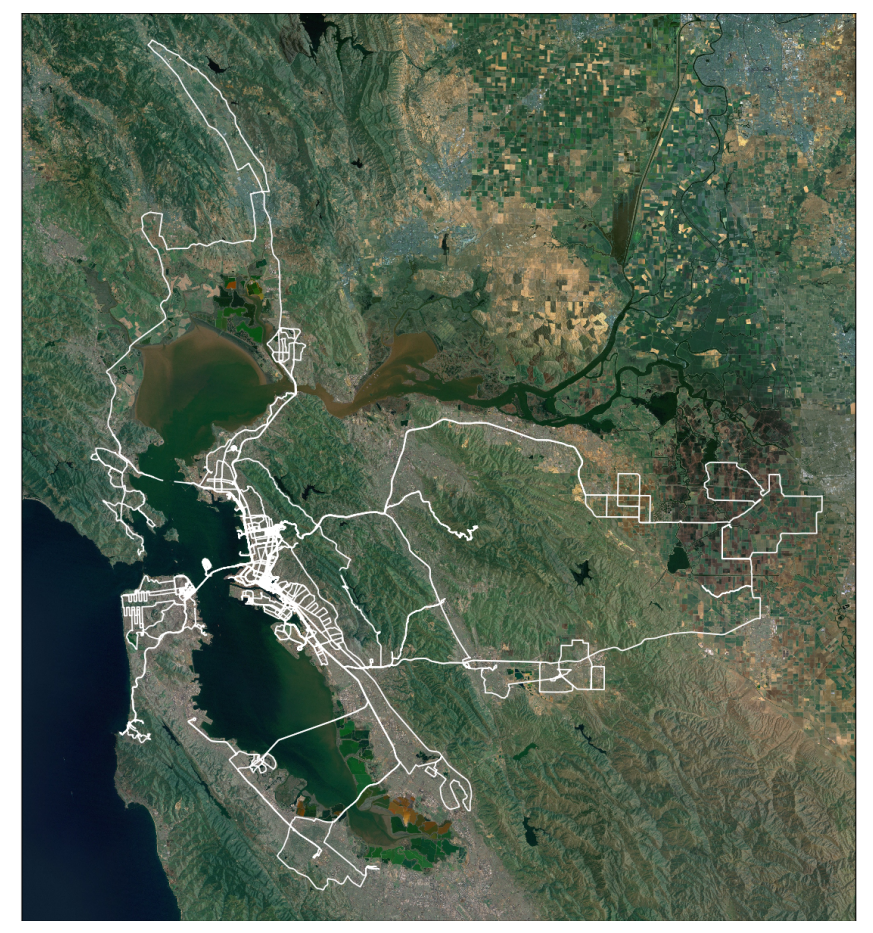

Figure 1: A map of RadMAP's surveys in the San Francisco Bay Area.

detector area and facing the right ("starboard") side of the vehicle. In between neighboring detectors is a gap of approximately $1.5 \mathrm{~cm}$. The detectors are read out by electronics described in Section 3.1.3. The typical energy resolution of the $\mathrm{NaI}$ detectors is $8.1 \%$ full width at half maximum (FWHM) at $662 \mathrm{keV}$. In front of the detectors is a coded mask that is 12 pixels high by 18 pixels wide. The mask has the same pitch as the detector array and is comprised of lead pixels measuring approximately 4 inch $\times 4$ inch $\times 2 \mathrm{~cm}$, which is the same length and width as the NaI detectors (Figure 3). The mask is positioned approximately $40 \mathrm{~cm}$ in front of the $\mathrm{NaI}$ array, yielding a pixel size of approximately $14^{\circ}$ square, a horizontal fully coded field of view of $90^{\circ}$, and a vertical fully coded field of view of $27^{\circ}$. The detector array is shielded around the sides and on the top by $2.5 \mathrm{~cm}$ of lead.

90 Two mask patterns have been used during RadMAP data collection thus far: 


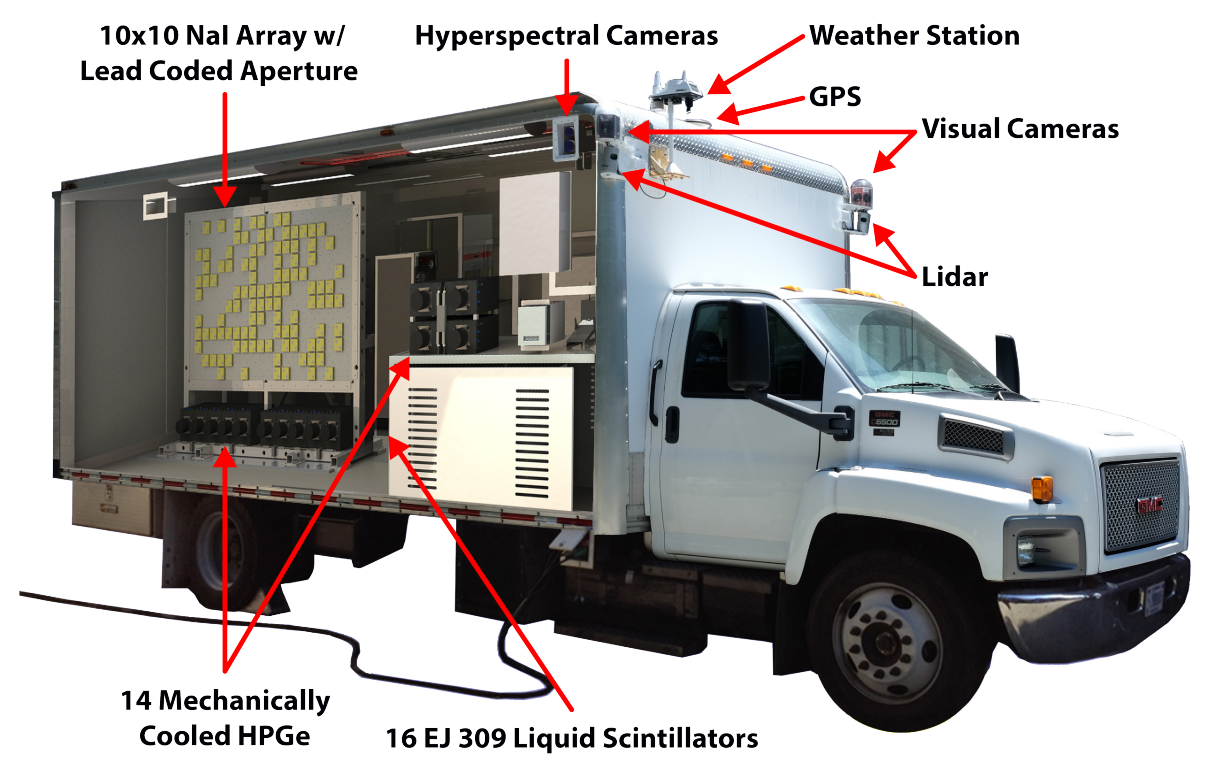

Figure 2: A cutaway view of the RadMAP truck showing the position of various radiation sensors. When there were between 18 and 24 liquid-nitrogen cooled HPGe detectors, additional detectors were mounted in a column to the left of the coded aperture.

the original coded mask pattern that the Naval Research Lab designed for the MISTI vehicle (the "NRL mask") and an improved random mask pattern (the "2015 mask"). Additionally, some data in 2013 were collected with no lead mask pieces in place, and some with a $10 \times 18$ version of the 2015 mask, in order to measure the effect of the amount of lead shielding on background variations [8]. Diagrams of both mask patterns are shown in Figure 3 . The 2015 mask pattern was found by attempting to suppress the image artifacts of the NRL mask, as well as by enforcing a condition that every $10 \times 10$ sub-array of the mask is nearly $50 \%$ transparent for a more uniform flat field image (Figure 44). Using balanced correlation to decode the masks also yields an improved system point spread function (SPSF), shown in Figure 5 [21, 22].

\subsubsection{HPGe array}

From 2011 to 2014, RadMAP fielded between 18 and 24 coaxial high purity germanium (HPGe) detectors manufactured by ORTEC. Each 100\% relative ef- 

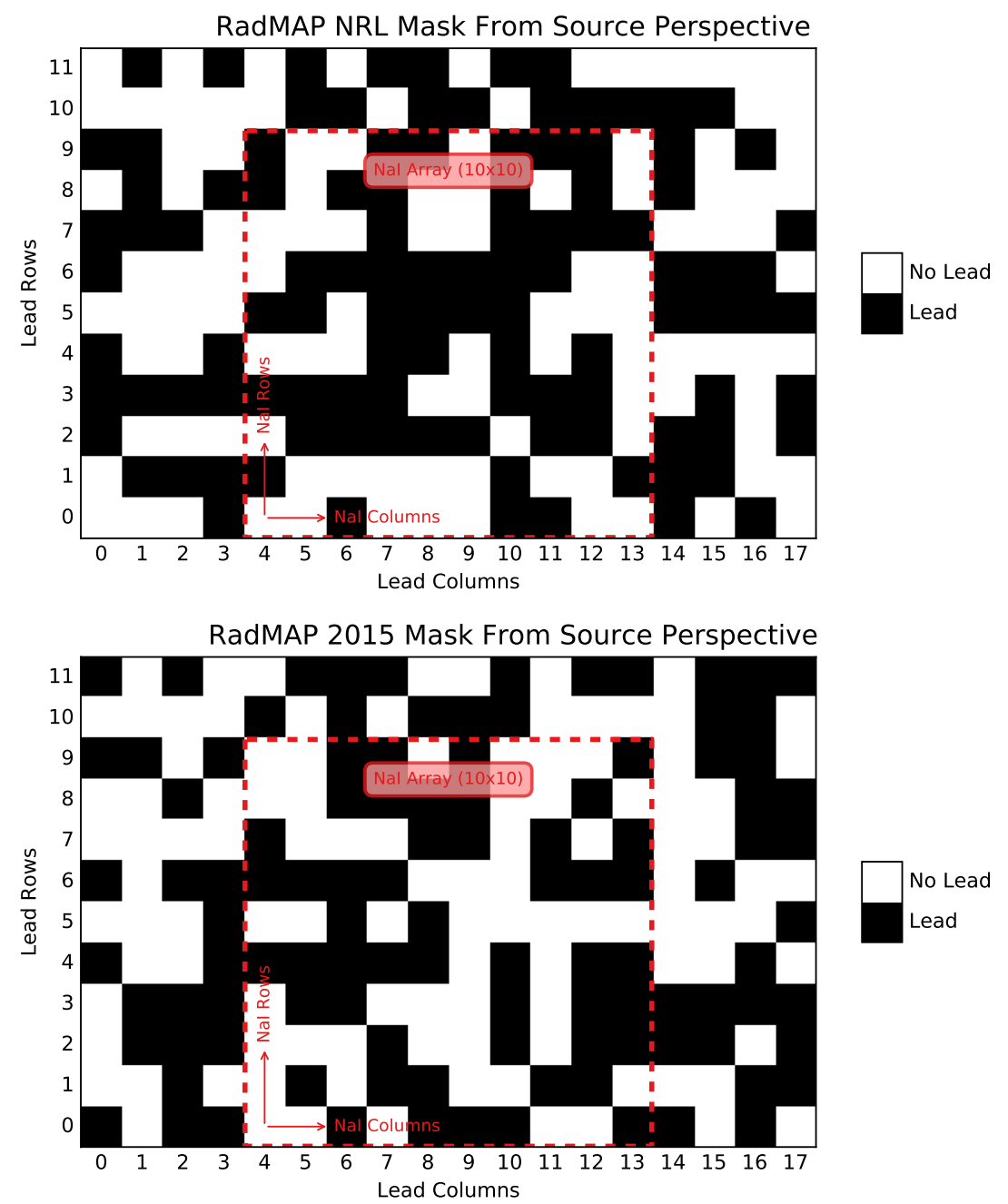

Figure 3: Original $18 \times 12$ coded mask design ("NRL mask," top) and the improved coded mask design ("2015 mask," bottom). The relative position of the $10 \times 10 \mathrm{NaI}$ detector array is outlined in red. The perspective is from the image plane looking back toward the vehicle.

ficiency detector was comprised of an approximately $88 \mathrm{~mm}$ diameter by $63 \mathrm{~mm}$ long germanium crystal and was cooled by an individual liquid-nitrogen dewar. The readout of the HPGe detectors was performed using the same electronics as the NaI array, although with different shaping times and higher analogto-digital converter (ADC) bit depth [20]. The typical energy resolution was 

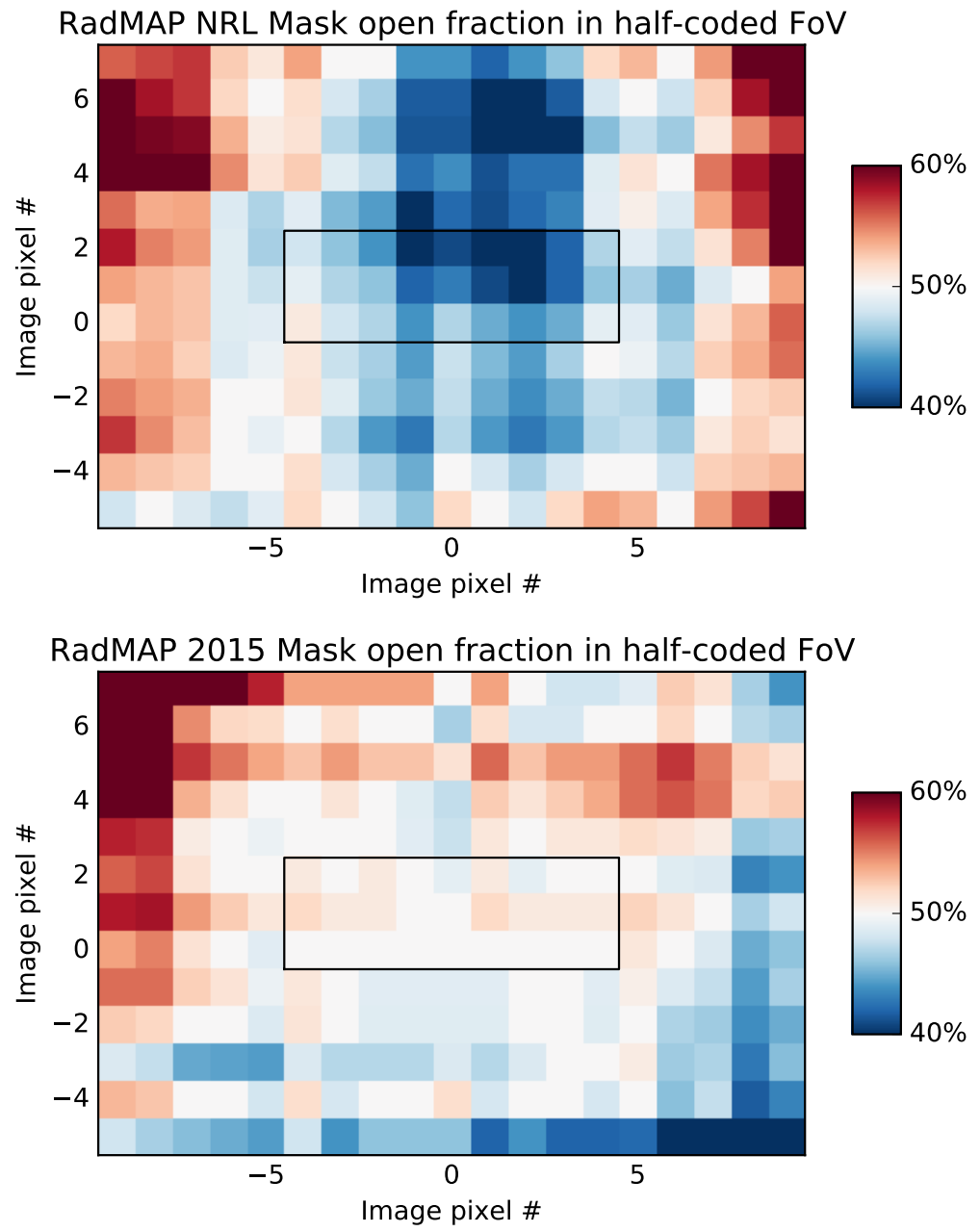

Figure 4: The open fraction of each mask in the half-coded field of view of the original and improved RadMAP coded masks. The 2015 mask shows a much more uniform flat field, especially in the fully coded field of view (outlined).

$1.7 \mathrm{keV}$ FWHM at $662 \mathrm{keV}$.

In the summer of 2015, the original liquid-nitrogen-cooled germanium detectors on RadMAP were replaced with 14 mechanically cooled IDM-200-V detector modules from ORTEC [23]. Each module contains a single $85 \mathrm{~mm}$ diameter by $30 \mathrm{~mm}$ long crystal cooled by a Stirling-cycle cooler. A custom BNC output 115 was added to each IDM for ease of integration with the existing readout system 

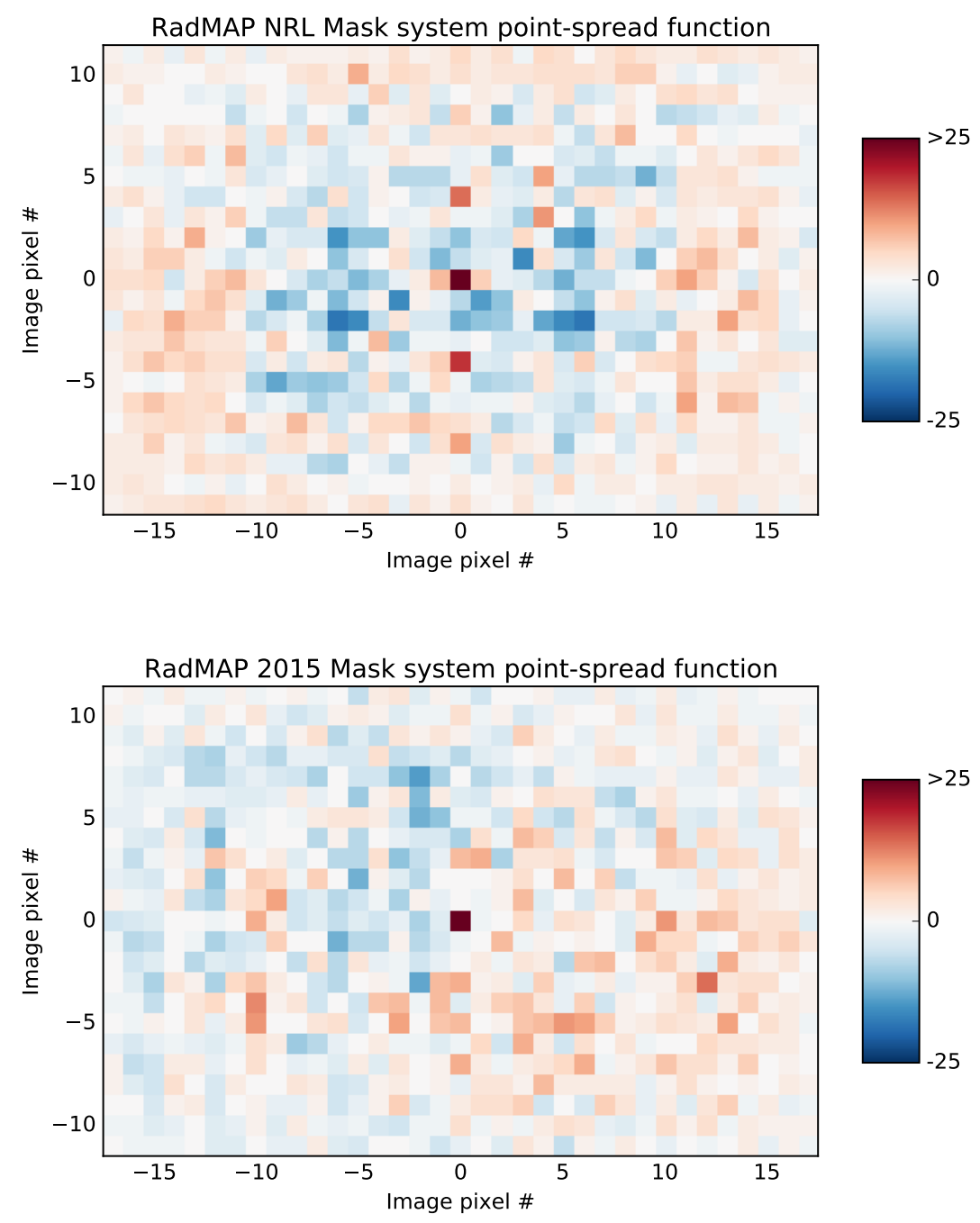

Figure 5: The system point-spread functions (SPSFs) of the original and improved RadMAP coded masks using balanced correlation to decode the mask [21, 22]. The ideal SPSF is a delta function with a maximum at $(0,0)$ whose value is the number of transparent pixels (in this case, 108). The 2015 mask shows improvement over the original mask by having smaller off-axis artifacts, both positive (correlated) and negative (anti-correlated) artifacts.

(see Section 3.1.3). 


\subsection{3. $\mathrm{NaI}(\mathrm{Tl})$ and $\mathrm{HPGe}$ readout}

From 2011-2014, the NaI(Tl) and HPGe detectors were read out by nine 16 channel Mesytec MSCF-16 shaping amplifier boards, which performed analog shaping of the detector signals, while five 32 channel Mesytec MADC-32 boards performed analog to digital conversion of the shaped signals [24, 25]. More details on the original readout system can be found in [20].

In 2015, in order to eliminate the dependency on the older analog hardware and eliminate a spurious noise signal that was observed in the HPGe detector data, the data acquisition system was upgraded to a fully digital system using eight Struck Innovative Systeme SIS3316 16-channel 14-bit $250 \mathrm{MHz}$ digitizers [26. These digitizers are individually read out over Ethernet by a dedicated Linux server.

The first SIS3316 board is synchronized using a $10 \mathrm{MHz}$ external NIM clock derived from the $10 \mathrm{MHz}$ sine output of the rubidium frequency standard (see Section 3.7 for details), and the remaining seven boards are synchronized to the first board using the LVDS cable bus on the front panel of the boards. In this way the boards are synchronized to the nanosecond level. The time synchronization of the boards was confirmed by searching the data for events where several detectors have events with timestamps within $10 \mathrm{~ns}$ of each other, and many muon events were identified as a result (Figure 6). Muon events could not be observed in the original analog readout system due to its lower dynamic range and timing resolution. Section 3.7 contains more information on the timing synchronization with other systems.

\subsubsection{Liquid scintillators}

Fast neutron signatures are valuable for the detection of special nuclear material (SNM). To explore the fast neutron domain with RadMAP, an array of liquid scintillators was installed. The scintillator array consists of 16 Eljen Technology EJ-309 organic liquid scintillators 27. Each detector is oriented horizontally and stacked vertically in two columns of eight detectors each. Each cell is a 5 inch diameter by 5 inch long aluminum cylinder coupled to a 5 inch 

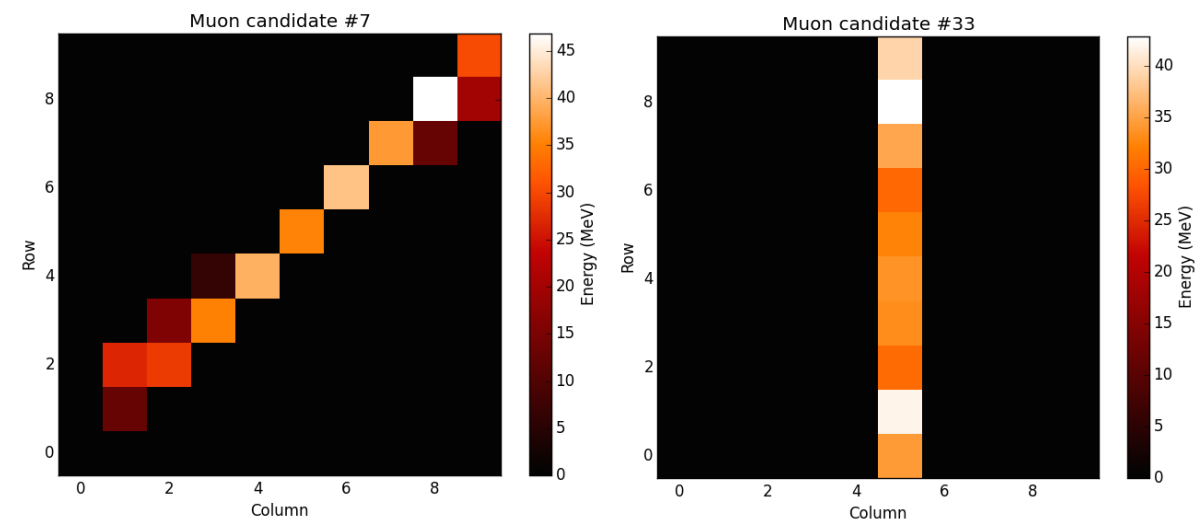

Figure 6: Two examples of muons detected by the NaI(Tl) array on board RadMAP. Muon events are found by searching for multi-detector events within 10 nanoseconds of each other, demonstrating the synchronization of the latest readout system.

Hamamatsu photomultiplier tube (PMT). The PMTs are powered by a VME high voltage power supply module controlled by iseg VHS 4030n (4 channels) and VHS C030n (12 channels) HV modules 28, which provide between $-1500 \mathrm{~V}$ and $-2000 \mathrm{~V}$ to each PMT. The total active detection volume of the system is approximately 25 liters. EJ-309 was selected for its pulse shape discrimination (PSD) characteristics, enabling neutron-induced signals to be distinguished from gamma-ray interactions [29, as well as for its low toxicity and high flash point.

The PMTs are read out by two Struck SIS3320 $250 \mathrm{MHz}$ 12-bit digitizers, each with eight channels. Each digitizer reads out one vertical column of eight detectors. The Struck-provided data acquisition software is used to control the digitizers and collect and save raw trace data. Once raw signal pulses with associated timing information are recorded, the data are then parsed into a usable format for data processing, PSD, and analysis. The data for the neutron detectors are saved on a dedicated laptop running Windows.

\subsection{RadMAP position and aspect}

Position information is provided by two systems. The first is a Magellan ADU5 GPS receiver with a $5 \mathrm{~Hz}$ data rate 30. This unit provides a coarse GPS location and the primary pulse-per-second (PPS) for the global clock. As this 
such as urban areas, where the GPS signal is attenuated or can be impacted by multipath effects (e.g., [31, 32]). Typical uncertainties range from $\sim 3$ meters in open areas to $\sim 10$ meters or more in urban areas. Because of these limitations, the use of the Magellan ADU5 on RadMAP was discontinued in 2015.

170 SPAN-CPT GPS/INS receiver 33. Because it uses inertial information as well as GPS information, its accuracy is greatly improved over the Magellan system, especially in urban areas. Typical accuracy is $3 \mathrm{~cm}$ after post-processing with public base station data as well as precise satellite orbit and clock information. accuracy. The NovAtel data is acquired over USB by the main Windows server A comparison between the Magellan ADU5 and the NovAtel SPAN-CPT for RadMAP data is shown in Figure 7 .

\section{3. $L i D A R$}

Two Velodyne HDL-32E LiDAR units 34 are installed on the left and right front corners of the truck (shown in Figure 2). Each LiDAR unit uses 32 infrared lasers to measure a three dimensional point cloud around the truck. The beam directions range from $+10.67^{\circ}$ to $-30.67^{\circ}$ in elevation, for an angular spacing of $1.33^{\circ}$. In azimuth, measurements are taken at an angular spacing of $0.12^{\circ}$ to $0.15^{\circ}$ as the LiDAR head rotates. The average rotation rate is approximately $10 \mathrm{~Hz}$, and the maximum range is $80-100 \mathrm{~m}$. In order to increase the vertical coverage of the LiDAR point clouds, each unit is tilted forward $10.67^{\circ}$ to cover $0^{\circ}$ to $-41.34^{\circ}$ in the forward direction and $+21.34^{\circ}$ to $-20^{\circ}$ in the rear direction. Custom acquisition code records the LiDAR data on RadMAP's main Windows 190 server.

\subsection{Visual imagery}

For wide field of view, high rate imagery, two Point Grey Ladybug 3 spherical video cameras [35] are installed at the top left and right corners of the truck cab, 

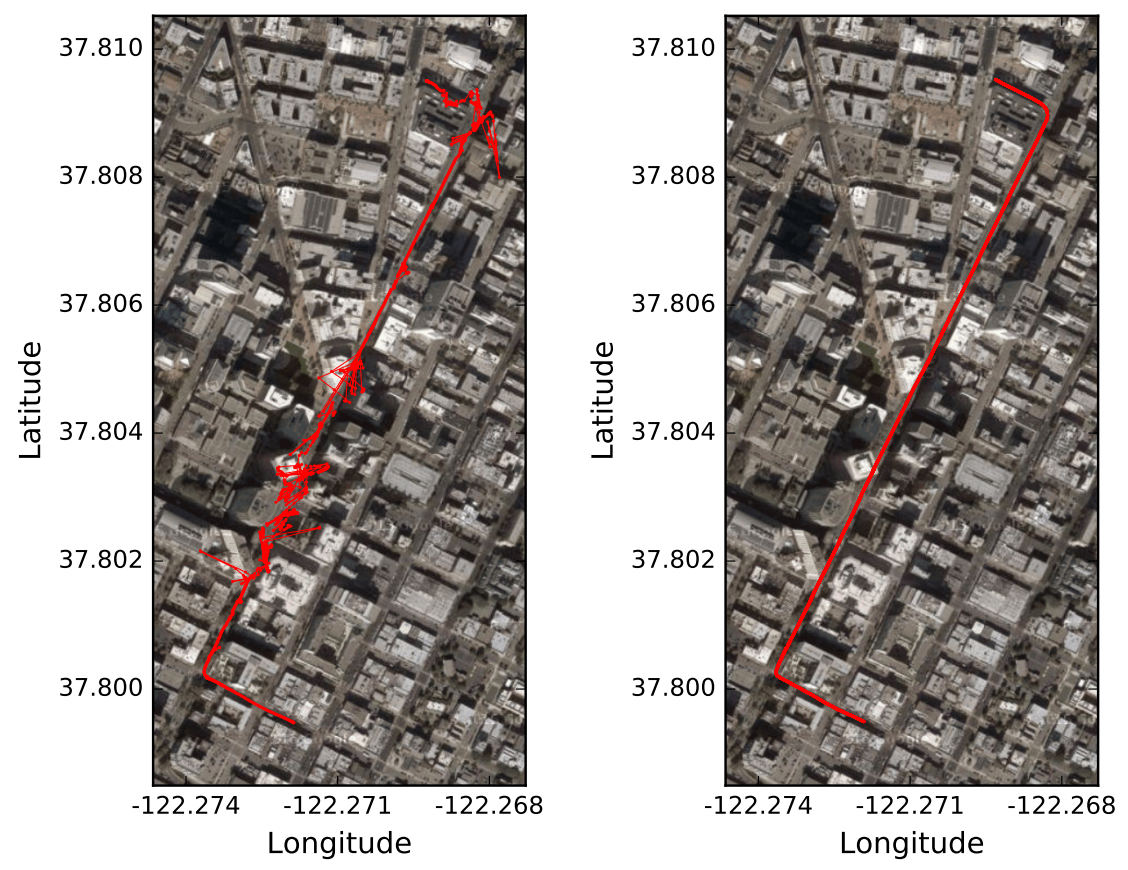

Figure 7: GPS locations from RadMAP in downtown Oakland using the Magellan ADU5 (left) and the NovAtel SPAN-CPT (right). The SPAN-CPT is able to remain highly accurate even when the view of the sky is blocked by tall buildings. (Google Maps imagery from DigitalGlobe, U.S. Geological Survey, and USDA Farm Service Agency.)

directly above the LiDAR sensors (Figure 2). Each camera uses six 2 megapixel sensors arranged to cover more than $80 \%$ of a full spherical field of view. Images are recorded at a rate of 15 frames per second. Custom acquisition code records data from both Ladybug cameras on the main Windows server.

In addition, a simple visual camera system covers the imaging field of view of the $\mathrm{NaI}(\mathrm{Tl})$ array. RadMAP currently uses a Raspberry Pi with a visual CCD camera and a fisheye lens, covering a field of view of approximately $100^{\circ}$ horizontal and $77^{\circ}$ vertical. This field of view subsumes the fully coded field of view of the gamma-ray imager. Images from this camera are recorded at a rate of $1 \mathrm{~Hz}$. In 2015, this camera replaced an older visual/infrared camera. 


\subsection{Weather}

205

New Mountain 100 recorded wind speed and direction, temperature, barometric pressure and relative humidity [36. Soon after, a standalone digital rain gauge was added to allow the study of correlations between rainfall and background level from radon. In December 2012, the weather station was upgraded to the computer.

\subsection{Sensor integration and synchronization}

In order to register data from all of the sensors, each computer and sensor records time in seconds since the UNIX epoch (i.e., midnight UTC on January 1, of reconstructed spatial datasets, e.g., in order to achieve an accuracy of $\lesssim 10 \mathrm{~cm}$ 
at typical driving speeds of $25 \mathrm{mph}(11 \mathrm{~m} / \mathrm{s})$, all data must be synchronized to $\lesssim 10 \mathrm{~ms}$.

In order to obtain the time accuracy required, the on-board computers are synchronized using Network Time Protocol (NTP) [40, which is capable of synchronizing the clocks to within a few milliseconds of the main clock. The computers are synchronized to the clock on the main Linux server, which is in turn synchronized to GPS time using the Magellan ADU5 and the utility GPSD [41. The Magellan outputs National Marine Electronics Association (NMEA) data messages and a pulse-per-second (PPS) signal, and together these are used to obtain a $\sim 1-\mu$ s-accuracy NTP time source. In addition, the PPS is stabilized by a Stanford Research Systems FS725 rubidium frequency standard 42 in case of a GPS dropout.

For some sensors, hardware triggering is preferred over software triggering in order to ensure that sufficient synchronization is maintained. For these sensors - the Ladybug cameras, the LiDARs, and the hyperspectral cameras - a system was developed to provide the required trigger pulse types and rates to each one. The hardware synchronization is controlled by an Arduino Mega 2560 [43], which receives both the NMEA string from the Magellan ADU5 as well as the PPS from the rubidium frequency standard. The Arduino is programmed to output the correct pulse frequency and shape to each of the sensors, e.g., a $5 \mathrm{~V}$ square wave at a frequency of $15 \mathrm{~Hz}$ for the Ladybug cameras, and these triggers are synchronized to the PPS pulse. The LiDARs require both a PPS trigger pulse and an NMEA message after a set delay.

In 2015, the NovAtel SPAN-CPT unit replaced the Magellan ADU5 as the source of both the NMEA messages and the PPS. Since the SPAN-CPT can be configured to output different NMEA messages, it was straightforward to use it as the main GPS time source for RadMAP. The new timing logic is shown in Figure 8 . 


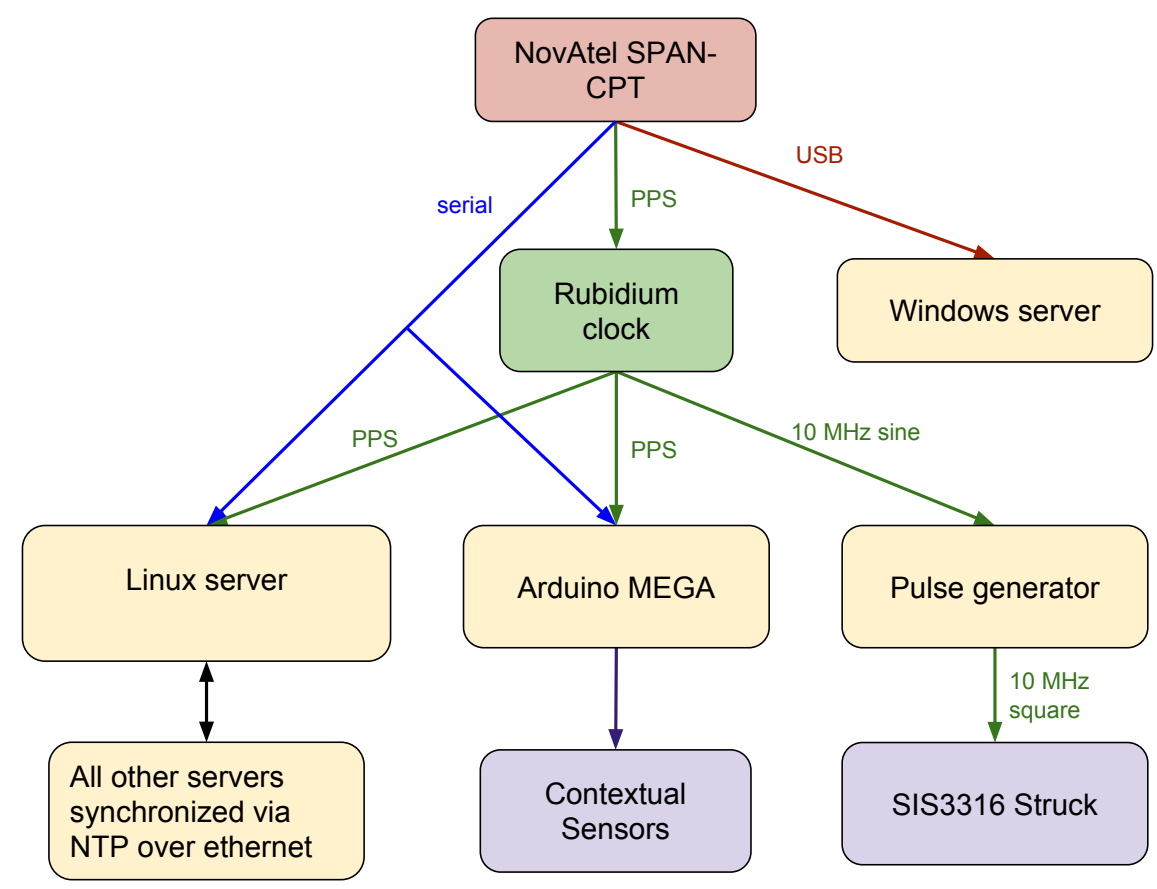

Figure 8: Diagram explaining how time is synchronized to high precision among the computer servers, SIS3316 boards, and contextual sensors on board RadMAP. Before 2015, the Magellan ADU5 was used instead of the NovAtel SPAN-CPT. The SPAN-CPT's high precision position and aspect information is logged over the USB connection to a Windows server.

\subsection{Data rates}

Data from all different sensors and detectors on board the RadMAP vehicle are stored on board on a redundant array of independent disks (RAID). Some of the sensors, e.g., the Ladybug cameras, have high data rates, while other sensors, such as the radiation detectors, typically have much lower data requirements. Table 1 shows the typical total data rate of the RadMAP system as well as a breakdown into the typical rates of the individual components.

\section{Data applications and use cases}

The large sets of synchronized, multi-sensor data acquired by RadMAP allow for the development and assessment of advanced detection and imaging algorithms, the investigation of novel data fusion approaches, and the exploration of 


\begin{tabular}{llc} 
Datastream & Format & Typical data rate (GB/hour) \\
\hline NaI and HPGe & list-mode & 3 \\
Liquid scintillators & list-mode with waveforms & 5 \\
Visual/IR camera & JPEG images & 0.5 \\
LiDARs (2) & libpcap format & 16 \\
Ladybug cameras (2) & PGR video format & 360 \\
Hyperspectral visual & ENVI files & 47 \\
Hyperspectral NIR & ENVI files & 25 \\
Other & Various & 0.5 \\
\hline Total & & $457 \mathrm{~GB} / \mathrm{hr}$
\end{tabular}

Table 1: The approximate amount of data collected per hour by each of the sensors on the RadMAP truck.

new concepts in mobile radiation detection. Already, the multi-sensor data collected with the RadMAP system have been used in a number of studies including an examination of the trade-offs between detection through spectroscopy and imaging [8], the characterization of an advanced spectroscopic detection algo275 rithm [44], and the development of techniques for the fusion of LiDAR, visual imagery, and radiation data (Section 4.3). Such studies are facilitated, in part, through the use of the Gamma-Ray Data Cloud (GRDC) data service for the curation, indexing, and querying of data (Section 4.6). In this section, we summarize several example use cases.

\subsection{Routine surveys}

Routine surveys of the San Francisco Bay Area have been performed with RadMAP since 2011 [7. Gamma-ray detector count rates for an urban region of the Bay Area (in this case, downtown Berkeley and Oakland) are shown in Figure 9. Increases in count rate are mostly due to passing particular building facades or going thorugh an underpass or tunnel, as well as encountering medical nuisance sources. These data demonstrate the non-Poissonian background distributions typically observed in an urban area.

In [7, the survey data were segmented into subsets based on environmental and geographic considerations. Individual datasets associated with urban, rural, 

turing data recorded on bridges. Analysis revealed that the local background distributions differed significantly both from each other and from the background distribution of all locations combined. This study served to illustrate the strong correlation between local environment and radiological background while suggesting that from an operational perspective, the use of context and/or environment specific detection thresholds and background models could allow for improved detection sensitivity at a constant false alarm rate.

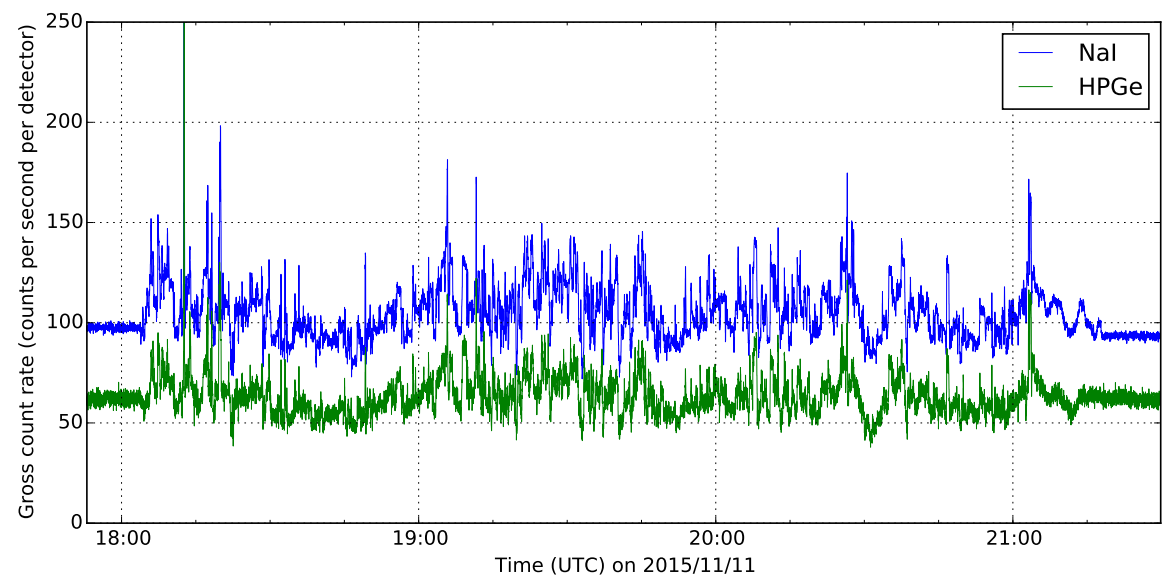

Figure 9: $\mathrm{NaI}(\mathrm{Tl})$ and HPGe gross count rates binned in 1-second intervals and normalized by number of detectors. The vehicle is stationary for the first 10 minutes and final 10 minutes of the survey, which is why count rates are flat there. The large increase in counts at 18:13 is due to an encounter with a medical source $\left({ }^{131} \mathrm{I}\right)$, and the maximum rate reaches 1800 counts per second per detector (truncated for display). There is also an encounter with a weak medical source $\left({ }^{99 m} \mathrm{Tc}\right)$ at 18:20, which is not obvious in comparison to the other fluctuations in the gross count rate but is clearly visible in the spectrum.

\subsection{Development and evaluation of gamma-ray detection algorithms}

The RadMAP dataset has also been used as a standard for characterizing and assessing the performance of gamma-ray detection algorithms. The dataset has been used to directly compare spectral- and imaging-based detection algorithms [8], evaluate the effects of detector efficiency and resolution using realistic backgrounds [45, and evaluate state-of-the-art detection and identification algorithms 44 . 
jection. Different spectral and imaging algorithms were applied to the data and compared against ideal cases. Even a simple linear region-of-interest spectral estimation was shown to offer a drastic improvement in false alarm rate over simple energy windowing. Likewise, the power of using imaging as a means to reduce systematic background fluctuations was also quantified. For the RadMAP imager, even simple spectroscopic approaches outperformed imaging approaches due to the higher number of source photons that would be detected without a mask.

In [45, RadMAP data were further applied to the study of the trade-offs between detector size and energy resolution. One of the key findings was that spectroscopic detection algorithms performed better than imaging methods for the RadMAP NaI $(\mathrm{Tl})$, but for a larger array or worse resolution detectors, imaging might eventually outperform spectroscopy. In addition, the HPGe detectors on RadMAP were compared to the $\mathrm{NaI}(\mathrm{Tl})$ array. It was found that when ${ }_{320}$ trained spectral algorithms are used, the equivalent of about seven $100 \%$ relative efficiency germanium detectors would yield the same performance as the $1 \mathrm{~m}^{2} \mathrm{NaI}(\mathrm{Tl})$ array, however these results are strongly dependent on the algorithm applied to the $\mathrm{NaI}$ data.

\subsection{Fusion of gamma-ray images, video, and 3D point clouds}

25 The use of real time LiDAR and video to augment the analysis of radiation data holds the potential to improve detection and localization performance while also providing contextually complete data products to aid in the interpretation of the radiation measurement. Potential concepts include the use of contextual cues present in visual imagery to inform detection algorithms of the local environment, the use of ranging information from LiDAR to improve lo-

In 8, data from RadMAP's $\mathrm{NaI}(\mathrm{Tl})$ array were analyzed using source incalization performance, as well as complete fusion of a gamma-ray image with a three-dimensional scene reconstruction [46, 47].

In one example from RadMAP data, panoramic imagery, LiDAR, and codedmask images have been fused into a video showing the location of a medical 
nuisance source $\left({ }^{99 m} \mathrm{Tc}\right)$ encountered in an urban area (Figure 10p. In this video, the coded-mask image was projected onto the LiDAR point cloud, which was in turn overlaid onto Ladybug imagery. The combined data product reveals that the source is in the front compartment of a nearby car. Fused data products such as this one could help rapidly identify and adjudicate sources in complex environments. A software pipeline for automatically producing data products like Figure 10 does not yet exist but is possible using existing data.

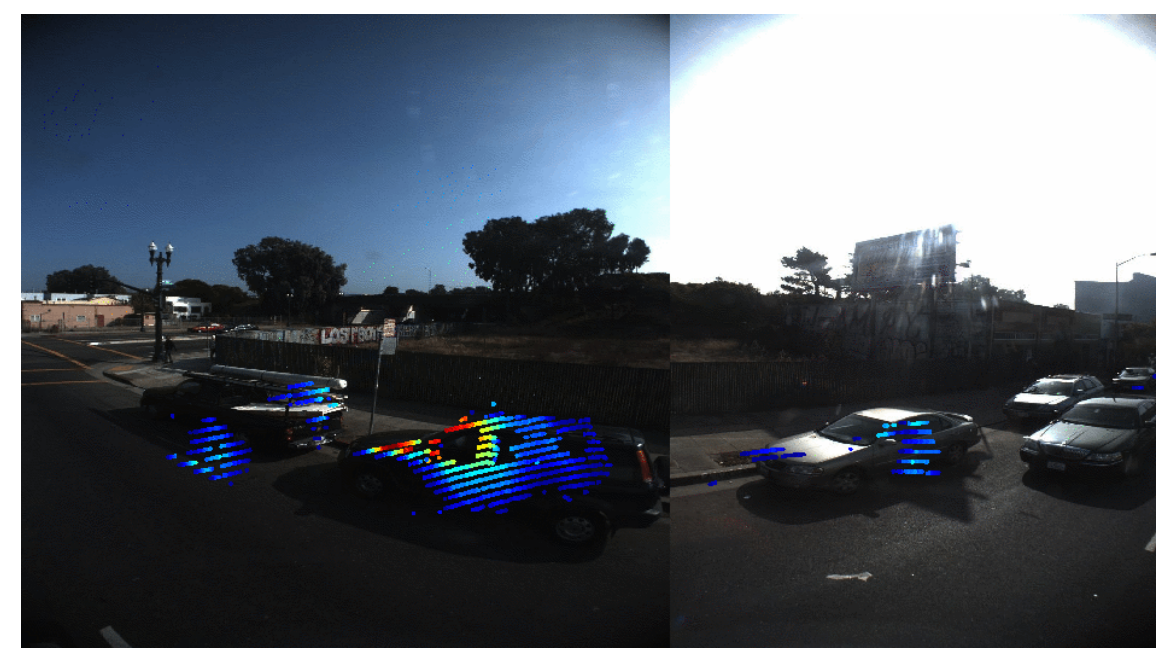

Figure 10: The fusion of a gamma-ray image of a medical isotope $\left({ }^{99 m} \mathrm{Tc}\right)$ using RadMAP's NaI array with a point cloud from a LiDAR, overlaid on visual imagery from RadMAP's Ladybug cameras. The source clearly appears in the driver's side of the SUV near the center of the image.

\subsection{Characterization of fast neutron background variability and correlations} with contextual data

Neutron backgrounds are primarily cosmogenic and are generally lower and less variable than gammas, but for weak SNM source detection, systematic variability still limits detection sensitivity. The largest known influence on neutron background variability is atmospheric pressure, which affects the development and attenuation of cosmic ray showers [48. Analysis of the RadMAP liquid scintillator data has confirmed that pressure is indeed the dominant factor in 

on-board weather station has been developed [49, 50].

After atmospheric pressure, the largest effect on the fast neutron background rate in urban areas has been found to be the "sky-view factor" [51]. The skyview factor is the fraction of the sky that is not blocked by nearby buildings,

which attenuate the cosmogenic neutrons. The resulting analysis of RadMAP fast neutron background data shows that, for sky-view factors $>0.15$, the shielding effect of buildings in urban environments is dominant over any additional production in building materials from spallation processes (Figure 11). Details and further discussion are in [49, 50,

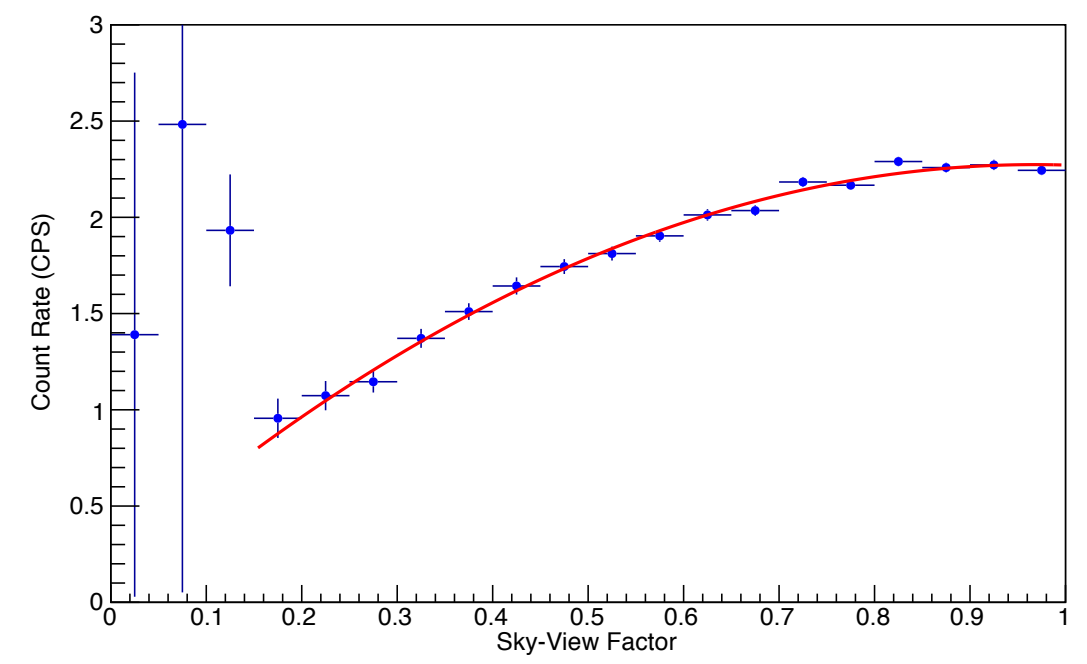

Figure 11: Pressure-adjusted count rates for sky-view factors from all runs that travel through select portions of Berkeley, Downtown Oakland, or Downtown San Francisco. A quadratic fit describes the data well for sky-view factors of 0.15 to 1.0. Low sky-view factors require further investigation and are excluded from the fit. From [49, 50.

\subsection{Hyperspectral image classification}

Hyperspectral imagers provide visual or infrared images with dozens to hundreds of channels. The additional spectral information can be used to identify 
the materials in a scene through identifying features in their reflectance spectra that are impossible to find using RGB imagery. Hyperspectral imagery has been used to distinguish different types of minerals, vegetation, soil, and manmade materials (e.g., [52, 53]). Since different types of materials often have very different radiological signatures, being able to easily distinguish different materials in a scene is a potentially powerful type of contextual data for radiation measurements that has been little examined to date.

An example of a largely unsupervised classification of HSI data into different materials is shown in Figure 12. The particular workflow used here was performed on data from RadMAP's NIR camera using the Environment for Visualizing Images (ENVI) software by Excelis [54]. The first step was to reduce the dimensionality of the data from 145 channels using the forward Minimum 375 Noise Fraction (MNF) rotation [55]. The MNF transform separates noise from data and orders the new MNF bands from low to high noise. The next step was to segment the scene; the first $30 \mathrm{MNF}$ bands were fed into a K-Means iterative spatial cluster classification method [56. This segmentation further reduces the dimensionality of the data before the final image classification step. In order to maintain adequate separation among spectrally dissimilar materials, 50 classes were chosen. The resulting image acts as an unsupervised Region of Interest (ROI) map. The final step is to convert the ROI map into a more relevant classified image. To do this we apply the Spectral Angle Mapper [57] method to the original 145 channel image using the ROI classes produced in the previous step. In this final step, spectrally similar ROI classes can be combined to form material classes. The end result is several material classes to represent the scene. This method resulted in the six readily interpreted classes for an urban scene in Figure 12 .

\subsection{Gamma-Ray Data Cloud}

The unique nature of the datasets produced by RadMAP are likely to be valuable to many research communities, including those engaged in detection algorithm development and computer vision research. To facilitate access to and 


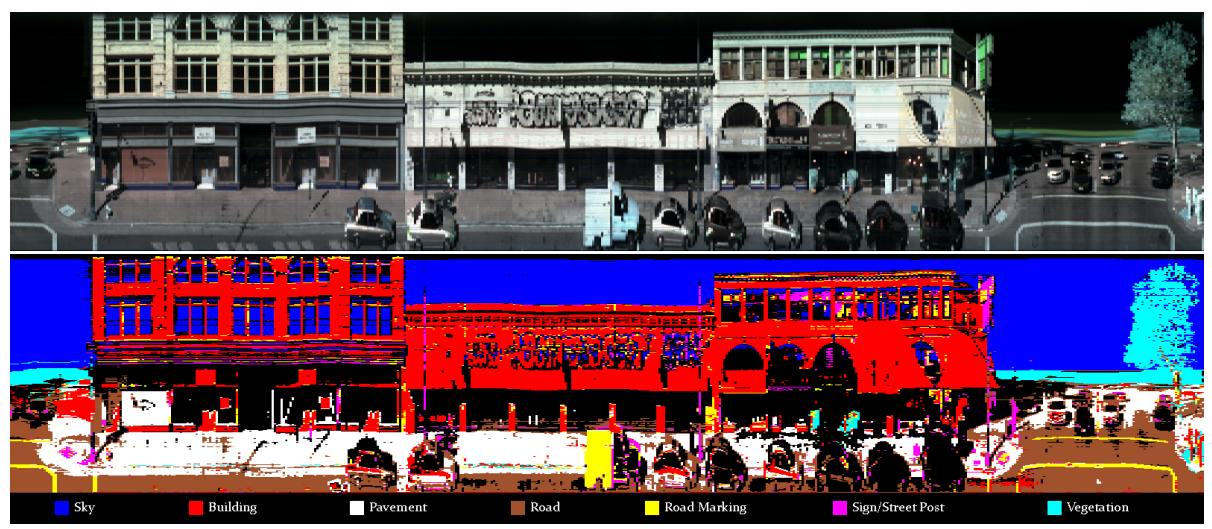

Figure 12: Demonstration of hyperspectral data being used to classify materials in an urban scene using data taken with the NIR camera on board RadMAP. At the top, a false color RGB image is generated using three Short-wave Infrared (SWIR) bands (1550, 1300, and $1050 \mathrm{~nm})$. The bottom image shows the six major classes that could be easily distinguished by a largely unsupervised classification pipeline (black pixels are unclassified). This material information could provide useful contextual information to associate with gamma-ray measurements.

analysis of RadMAP's synchronized datasets by collaborators, a data service has been developed called the Gamma-Ray Data Cloud (GRDC) [58, 59.

The data service contains datasets from the RadMAP detectors that are registered by timestamp and provides a web frontend for the browsing of available datasets and the formation of queries of the data (Figure 13). Front-end visualization tools include colorizing one-dimensional RadMAP paths by any available one-dimensional dataset (GPS-measured altitude is shown in Figure 13), creating histograms of one-dimensional datasets, and creating two-dimensional histograms of any time-synchronized list-mode dataset.

Queries consist of the desired datasets as well as any selections by time, latitude and longitude, or any other one-dimensional dataset (the latter selections are generated using the histogram tool). Web requests for data result in automated generation of requested data into an HDF5 file 60, which is made available for download. The data service provides an API for querying and downloading sequential portions of the datasets. The API also supports the creation of new, user-defined datasets that are registered back into the data service and can be made available to other users of the system for comparison 

allowing for broader access within the community and a common framework in which to register, share, and compare results. Such a large, shared dataset can 


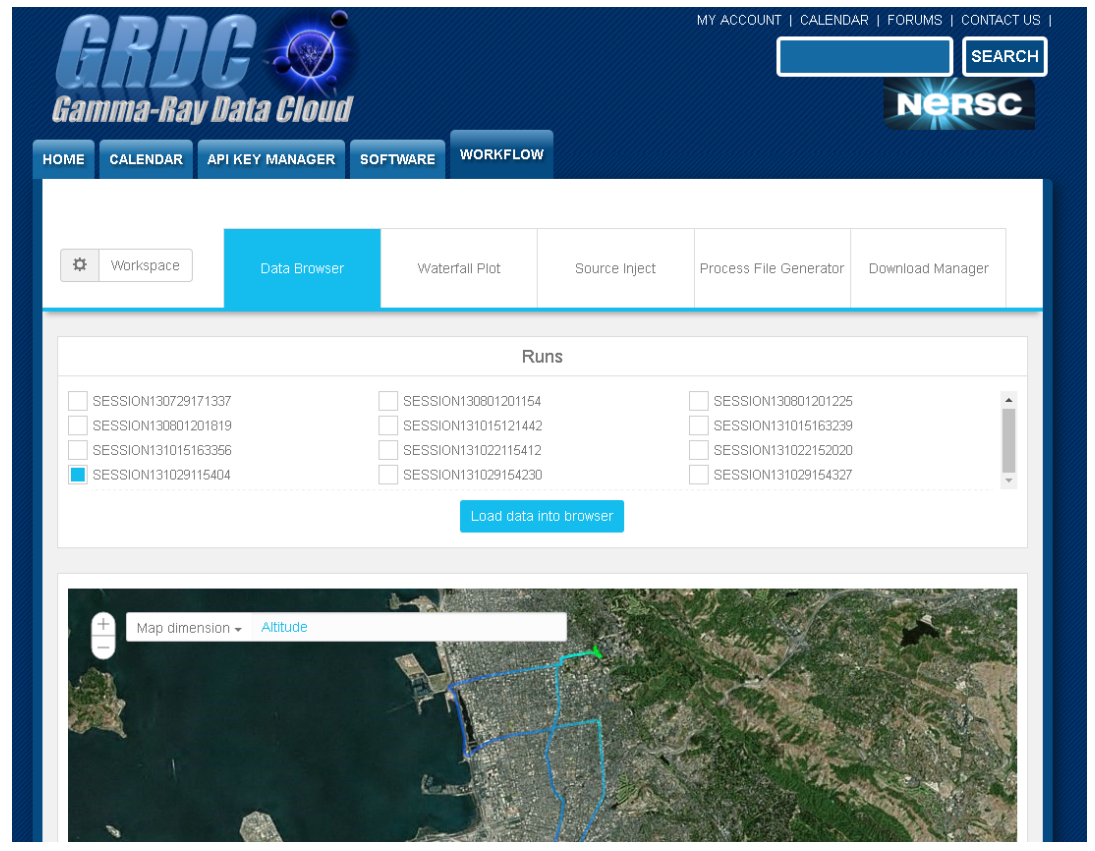

Figure 13: The GRDC web frontend, which allows for browsing, querying, and downloading different datasets from the RadMAP vehicle, as well as the creation of new, user-defined datasets.

enable new collaborative developments in detector modalities and algorithms for homeland security, new concepts in multi-sensor data fusion, and a more complete understanding of complex urban gamma-ray backgrounds.

\section{Acknowledgments}

This work was supported by the U.S. Department of Homeland Security Domestic Nuclear Detection Office under Grant Award 2011-DN-077-ARI04903, HSHQDC-14-X-00197, and IAA HSHQDC-11-X-00380.

This work was also performed under the auspices of the U. S. Department of Energy by Lawrence Berkeley National Laboratory under Contract DE-AC0205 CH11231.

Data storage, processing, and the GRDC science gateway all use resources of the National Energy Research Scientific Computing Center, which is sup- 
ported by the Office of Science of the U.S. Department of Energy under Contract No. DE-AC02-05CH11231. This support does not constitute an express or implied endorsement on the part of the government.

The authors would like to thank Luster Howard, Shelley Caras, Robert Kelley, Paul Williams, and Jon Cleveland from Lawrence Berkeley Lab Facilities for driving the RadMAP vehicle. We would also like to thank Michael Johnson, Paul Fallon, Timothy Loew, Joseph Wallig, Brien Ninemire, Tom Perry, John Garcia, Steve Warner, Michel Kireeff Covo, and the rest of the staff of Building 88 for providing excellent support. The authors also thank the team from the Naval Research Lab for designing, building, and providing training and support for the original MISTI system. Thanks also to Erika Suzuki for proofreading this document.

\section{References}

[1] K.-P. Ziock, M. Cunningham, L. Fabris, Two-sided coded-aperture imaging without a detector plane, in: IEEE Nuclear Science Symposium Conference Record, 2008. NSS '08, 2008, pp. 634-641. doi:10.1109/NSSMIC.2008. 4775216

[2] E. A. Wulf, B. F. Phlips, W. Neil Johnson, B. Leas, L. J. Mitchell, MISTI imaging and source localization, in: IEEE Nuclear Science Symposium Conference Record, 2008. NSS '08, 2008, pp. 2413-2417. doi:10.1109/ NSSMIC.2008.4774842,

[3] R. D. Penny, W. E. Hood, R. M. Polichar, F. H. Cardone, L. G. Chavez, S. G. Grubbs, B. P. Huntley, R. A. Kuharski, R. T. Shyffer, L. Fabris, K. P. Ziock, S. E. Labov, K. Nelson, A dual-sided coded-aperture radiation detection system, Nuclear Instruments and Methods in Physics Research Section A: Accelerators, Spectrometers, Detectors and Associated Equipment 652 (1) (2011) 578-581. doi:10.1016/j.nima.2011.01.161.

口 URL http://ww.sciencedirect.com/science/article/pii/ S0168900211002634 

D. McDevitt, M. Wittbrodt, B. Milbrath, SORISA standoff radiation imaging system, Nuclear Instruments and Methods in Physics Research Section A: Accelerators, Spectrometers, Detectors and Associated Equipment 652 (1) (2011) 5-9. doi:10.1016/j.nima.2011.02.068

[5] K.-P. Ziock, J. Collins, W. Craig, L. Fabris, R. Lanza, S. Gallagher, B. K. P. Horn, N. Madden, E. Smith, M. Woodring, Source-search sensitivity of a large-area, coded-aperture, gamma-ray imager, in: 2004 IEEE Nuclear Science Symposium Conference Record, Vol. 1, 2004, pp. 322-326 Vol. 1. doi:10.1109/NSSMIC.2004.1462205

口 [6] K.-P. Ziock, K. E. Nelson, Maximum detector sizes required for orphan source detection, Nuclear Instruments and Methods in Physics Research Section A: Accelerators, Spectrometers, Detectors and Associated Equipment 579 (1) (2007) 357-362. doi:10.1016/j.nima.2007.04.061.

[7] T. Aucott, M. Bandstra, V. Negut, D. Chivers, R. Cooper, K. Vetter, Routine Surveys for Gamma-Ray Background Characterization, IEEE Transactions on Nuclear Science 60 (2) (2013) 1147-1150. doi:10.1109/TNS. 2013.2251355

[8] T. Aucott, M. Bandstra, V. Negut, J. Curtis, D. Chivers, K. Vetter, Effects of Background on Gamma-Ray Detection for Mobile Spectroscopy and Imaging Systems, IEEE Transactions on Nuclear Science 61 (2) (2014) 985-991. doi:10.1109/TNS.2014.2306998.

q [9] H. K. Aage, U. Korsbech, Search for lost or orphan radioactive sources based on NaI gamma spectrometry, Applied Radiation and Isotopes 58 (1) (2003) 103-113. doi:10.1016/S0969-8043(02)00222-1. 
URL http://www.sciencedirect.com/science/article/pii/ S0969804302002221

[10] S. Labov, L. Pleasance, W. Sokkappa, W. Craig, G. Chapline, M. Frank, J. Gronberg, J. Jernigan, S. Johnson, J. Kammeraad, D. Lange, A. Meyer, K. Nelson, B. Pohl, D. Wright, R. Wurtz, Foundations for Improvements to Passive Detection Systems - Final Report, UCRL-TR-207129doi:10. 2172/15011571.

[11] K. D. Jarman, R. C. Runkle, K. K. Anderson, D. M. Pfund, A comparison 1 of simple algorithms for gamma-ray spectrometers in radioactive source search applications, Applied Radiation and Isotopes 66 (3) (2008) 362-371. doi:10.1016/j.apradiso.2007.09.010. URL http://ww.sciencedirect.com/science/article/pii/ S0969804307002886

[12] K. K. Anderson, K. D. Jarman, M. L. Mann, D. M. Pfund, R. C. 口 Runkle, Discriminating nuclear threats from benign sources in gammaray spectra using a spectral comparison ratio method, Journal of Radioanalytical and Nuclear Chemistry 276 (3) (2008) 713-718. doi:10.1007/s10967-008-0622-x.

口 URL http://link.springer.com/article/10.1007/ s10967-008-0622-x

[13] T. Burr, M. Hamada, Radio-Isotope Identification Algorithms for NaI Spectra, Algorithms 2 (1) (2009) 339-360. doi:10.3390/a2010339.

URL http: //www .mdpi . com/1999-4893/2/1/339

[14] D. Pfund, K. Jarman, B. Milbrath, S. Kiff, D. Sidor, Low Count Anomaly Detection at Large Standoff Distances, IEEE Transactions on Nuclear Science 57 (1) (2010) 309-316. doi:10.1109/TNS.2009.2035805

${ }_{535}[15]$ D. K. Fagan, S. M. Robinson, R. C. Runkle, Statistical methods a applied to gamma-ray spectroscopy algorithms in nuclear security 
missions, Applied Radiation and Isotopes 70 (10) (2012) 2428-2439. doi:10.1016/j.apradiso.2012.06.016.

[16] B. R. Cosofret, K. Shokhirev, P. Mulhall, D. Payne, B. Harris, Utilization

1 of advanced clutter suppression algorithms for improved standoff detection and identification of radionuclide threats, in: Proceedings of SPIE, Vol. 9073, 2014, pp. 907316-907316-13. doi:10.1117/12.2049831. URL http://dx.doi.org/10.1117/12.2049831

[17] R. S. Detwiler, D. M. Pfund, M. J. Myjak, J. A. Kulisek, C. E. Seifert, घ Spectral anomaly methods for aerial detection using KUT nuisance rejection, Nuclear Instruments and Methods in Physics Research Section A: Accelerators, Spectrometers, Detectors and Associated Equipment 784 (2015) 339-345. doi:10.1016/j.nima.2015.01.040. URL http://www.sciencedirect.com/science/article/pii/ S0168900215000716

[18] K.-P. Ziock, W. Craig, L. Fabris, R. Lanza, S. Gallagher, B. K. P. Horn, N. Madden, Large area imaging detector for long-range, passive detection of fissile material, IEEE Transactions on Nuclear Science 51 (5) (2004) 2238-2244. doi:10.1109/TNS.2004.836117

[19] K.-P. Ziock, L. Fabris, D. Carr, J. Collins, M. Cunningham, F. Habte, T. Karnowski, W. Marchant, A fieldable-prototype, large-area, gamma-ray imager for orphan source search, in: IEEE Nuclear Science Symposium Conference Record, 2007. NSS '07, Vol. 2, 2007, pp. 949-958. doi:10. 1109/NSSMIC. 2007.4437175

[20] L. Mitchell, B. F. Phlips, W. Johnson, E. A. Wulf, A. Hutcheson, C. Lister, K. Bynum, B. Leas, G. Guadagno, Mobile imaging and Spectroscopic Threat Identification (MISTI): System overview, in: 2009 IEEE Nuclear 
Science Symposium Conference Record (NSS/MIC), 2009, pp. 110-118. doi:10.1109/NSSMIC.2009.5401849

[21] E. E. Fenimore, T. M. Cannon, Coded aperture imaging with uniformly redundant arrays, Applied Optics 17 (3) (1978) 337. doi:10.1364/A0.17.000337 http://www.opticsinfobase.org/abstract.cfm?URI= ao-17-3-337

[22] E. Caroli, J. B. Stephen, G. D. Cocco, L. Natalucci, A. Spizzichino, Coded aperture imaging in X- and gamma-ray astronomy, Space Science Reviews 45 (3-4) (1987) 349-403. doi:10.1007/BF00171998 URL http://link.springer. com/article/10.1007/BF00171998

[23] ORTEC, IDM-200-V - Ortec URL http://www . ortec-online.com/download/IDM-200-V.pdf

[24] mesytec GmbH, MSCF-16 URL http://www.mesytec.com/mscf.html

${ }_{580}$ [25] mesytec GmbH, MADC-32.

URL http://www.mesytec.com/datasheets/MADC-32.pdf

[26] Struck Innovative Systeme, SIS3316 16 channel 125/250 MSPS 14/16-bit Digitizer Family

URL http://www.struck.de/sis3316.html

[27] Eljen Technology, EJ-309.

a URL http://www.eljentechnology.com/index.php/products/ liquid-scintillators/73-ej-309

[28] iseg Spezialelektronik GmbH, VHS - Versatile High Voltage Module in VME Standard.

URL http://iseg-hv.com/en/products/detail/VHS 
[29] L. Swiderski, M. Moszyski, D. Wolski, J. Iwanowska, T. Szczniak,

п P. Schotanus, C. Hurlbut, Suppression of gamma-ray sensitivity of liquid scintillators for neutron detection, Nuclear Instruments and Methods in Physics Research Section A: Accelerators, Spectrometers, Detectors and Associated Equipment 652 (1) (2011) 330-333. doi:10.1016/j.nima.2011.01.115

口 URL http://www.sciencedirect.com/science/article/pii/ S0168900211002014

[30] Magellan Navigation Inc., ADU5 - Operation \& Reference Manual (2007). URL ftp://ftp.ashtech.com/OEM_Sensor_ADU/Legacy\%20products/ ADU5/Reference\%20Material/ADU5\%200RM\%20Rev\%20C.pdf

[31] J. i. Meguro, T. Murata, J. i. Takiguchi, Y. Amano, T. Hashizume, GPS Multipath Mitigation for Urban Area Using Omnidirectional Infrared Camera, IEEE Transactions on Intelligent Transportation Systems 10 (1) (2009) 22-30. doi:10.1109/TITS.2008.2011688.

[32] M. Obst, S. Bauer, G. Wanielik, Urban multipath detection and mitigation with dynamic 3d maps for reliable land vehicle localization, in: Position Location and Navigation Symposium (PLANS), 2012 IEEE/ION, 2012, pp. 685-691. doi:10.1109/PLANS.2012.6236944

${ }_{610}^{63}$ [3] NovAtel Inc., SPAN-CPT Single Enclosure GNSS/INS Receiver. URL http://www.novatel.com/products/ span-gnss-inertial-systems/span-combined-systems/span-cpt/

[34] Velodyne LiDAR, HDL-32E.

URL http://velodynelidar.com/hdl-32e.html

${ }_{615}$ [35] Point Grey Research, Ladybug3 1394b (2015). URL https://www.ptgrey.com/ladybug3-360-degree-firewire-spherical-camera-systems

[36] New Mountain Innovations, Ultrasonic Weather Station (2015). URL http://www.newmountain.com/acoustic-weather-station/ 
[37] Davis Instruments Corp., Vantage Vue Wireless Weather Station (2015).

[38] Resonon, Pika II - VNIR Hyperspectral Imaging Camera.

URL http://www.resonon.com/Products/imagers_pika_ii.html

[39] Resonon, Pika NIR - Near Infrared Hyperspectral Imaging Camera.

URL http://www.resonon.com/Products/imagers_pika_nir.html

[40] NTP Project, ntp.org: Home of the Network Time Protocol (2015).

URL http://www.ntp.org

[41] GPSD Project, gpsd a GPS service daemon (2015).

URL http://www . catb.org/gpsd/

[42] Stanford Research Systems, Rubidium Frequency Standard - FS725.

URL http://www.thinksrs . com/products/FS725.htm

[43] Arduino, Arduino - ArduinoBoardMega2560.

URL https://www . arduino.cc/en/Main/arduinoBoardMega2560

[44] T. Joshi, R. Cooper, J. Curtis, M. Bandstra, B. Cosofret, K. Shokhirev, D. Konno, A Comparison of the Detection Sensitivity of the Poisson Clutter Split and Region of Interest Algorithms on the RadMAP Mobile System, IEEE Transactions on Nuclear Science 63 (2). doi:10.1109/TNS.2016. 2537206

[45] T. J. Aucott, M. S. Bandstra, V. Negut, J. C. Curtis, R. E. Meyer, D. H. Chivers, K. Vetter, Impact of detector efficiency and energy resolution on gamma-ray background rejection in mobile spectroscopy and imaging systems, Nuclear Instruments and Methods in Physics Research Section A: Accelerators, Spectrometers, Detectors and Associated Equipment 789 (2015) 128-133. doi:10.1016/j.nima.2015.04.012.

URL http://www.sciencedirect.com/science/article/pii/ 
[46] L. Mihailescu, K. Vetter, D. Chivers, Standoff 3d Gamma-Ray Imaging, IEEE Transactions on Nuclear Science 56 (2) (2009) 479-486. doi:10. 1109/TNS.2009.2015304.

[47] R. Barnowski, A. Haefner, L. Mihailescu, K. Vetter, Scene data fusion: Real-time standoff volumetric gamma-ray imaging, Nuclear Instruments and Methods in Physics Research Section A: Accelerators, Spectrometers, Detectors and Associated Equipment 800 (2015) 65-69. doi:10.1016/j.nima.2015.08.016

घ URL http://www.sciencedirect.com/science/article/pii/ S016890021500950X

[48] J. F. Ziegler, Terrestrial cosmic rays, IBM Journal of Research and Development 40 (1) (1996) 19-39. doi:10.1147/rd.401.0019.

[49] J. Davis, Liquid Scintillator Detectors for Fast Neutron Background Characterization with the Radiological Multi-sensor Analysis Platform (RadMAP), Master's thesis, University of California, Berkeley (2015).

[50] J. Davis, E. Brubaker, K. Vetter, Fast neutron background characterization with the Radiological Multi-sensor Analysis Platform (RadMAP) (in preparation).

[51] A. Iyengar, M. Beach, R. J. Newby, L. Fabris, L. H. Heilbronn, J. P. Hayward, Systematic measurement of fast neutron background fluctuations in an urban area using a mobile detection system, Nuclear Instruments and Methods in Physics Research Section A: Accelerators, Spectrometers, Detectors and Associated Equipment 773 (2015) 27-32. doi:10.1016/j.nima.2014.10.045 URL http://www.sciencedirect.com/science/article/pii/ S0168900214012029

[52] M. B. Satterwhite, J. P. Henley, Hyperspectral Signatures (400 to $2500 \mathrm{~nm}$ ) of Vegetation, Minerals, Soils, Rocks, and Cultural Features: Laboratory and Field Measurements, Tech. rep. (Dec. 1990). 
680

[54] Exelis Visual Information Solutions, ENVI (2015). ENVI.aspx

[55] A. Green, M. Berman, P. Switzer, M. Craig, A transformation for ordering

[57] F. A. Kruse, A. B. Lefkoff, J. W. Boardman, K. B. Heidebrecht, A. T.

[58] Lawrence Berkeley National Lab, Gamma-Ray Data Cloud (2013).

URL https://grdc.nersc.gov multispectral data in terms of image quality with implications for noise removal, IEEE Transactions on Geoscience and Remote Sensing 26 (1) (1988) 65-74. doi:10.1109/36.3001.

[56] J. T. Tou, R. C. Gonzalez, Pattern recognition principles, Pattern Recognition in Physics -1.

$$
\text { URL http://adsabs.harvard.edu/abs/1974prp. .book ..... T }
$$

Shapiro, P. J. Barloon, A. F. H. Goetz, The spectral image processing

system (SIPS)interactive visualization and analysis of imaging spectrometer data, Remote Sensing of Environment 44 (23) (1993) 145-163. doi : 10.1016/0034-4257(93)90013-N.

URL http://www.sciencedirect.com/science/article/pii/ $003442579390013 \mathrm{~N}$

[59] B. Quiter, L. Ramakrishnan, M. Bandstra, GRDC: a Collaborative Framework for Radiological Background and Contextual Data Analysis: Project: LB11-SAM Feasibility Study-PD3SJ, Tech. Rep. LBNL-1003947, Lawrence Berkeley National Laboratory (2015). 
[60] The HDF Group, Hierarchical Data Format, version 5, http://www.hdfgroup.org/HDF5/ (1997-2016). 